\title{
ON THE PERIODIC NATURE OF SOME MAX-TYPE DIFFERENCE EQUATIONS
}

\author{
E. M. ELABBASY, H. EL-METWALLY, AND E. M. ELSAYED \\ Received 10 April 2005 and in revised form 19 June 2005
}

We study some qualitative behavior of solutions of some max-type difference equations with periodic coefficients. Some new results of the periodicity character of solutions of that type of difference equations will be established.

\section{Introduction}

Recently there has been a lot of interest in studying the global attractivity, the boundedness character, and the periodicity nature of nonlinear difference equations. In $[5,6,8]$ some global convergence results were established which can be applied to nonlinear difference equations in proving that every solution of these equations converges to a periodic solution (which need not necessarily be stable). The periodic nature of nonlinear difference equations of the max type has been investigated by many authors. See for example $[1,2,3,4]$.

Our main objective in this paper is to extend the study of boundedness and periodicity to solutions of some max-type difference equations. We deal with the following difference equation:

$$
x_{n+1}=\max \left\{\frac{1}{x_{n}}, \frac{A_{n}}{x_{n-1}}\right\}, \quad n=0,1, \ldots
$$

where $\left\{A_{n}\right\}_{n=0}^{\infty}=\{\ldots, \alpha, \beta, \alpha, \beta, \ldots\}$ is a periodic sequence of positive numbers of period two with $\beta>\alpha>1$. The case where $\left\{A_{n}\right\}_{n=0}^{\infty}$ is a periodic sequence of positive numbers of period three and $A_{n} \in(0,1]$ was investigated in [4].

\section{Invariant interval and boundedness}

In this section, we show that every solution of (1.1) is bounded and persists.

The following lemmas are quite important results in their own; however these lemmas will be used in the subsequent discussion.

Lemma 2.1. Every positive solution of (1.1) is bounded and persists. 
2228 Periodicity of max-type difference equations

Proof. Let $\left\{x_{n}\right\}_{n=-1}^{\infty}$ be a solution of (1.1). It follows from (1.1) for an integer number $N \geq 0$ that

$$
x_{n+1} x_{n} \geq 1, \quad x_{n+1} x_{n-1} \geq \alpha>1 \quad \forall n \geq N .
$$

Thus

$$
\min \left\{x_{n+1} x_{n}, x_{n+1} x_{n-1}\right\} \geq 1
$$

or

$$
x_{n+1} \min \left\{x_{n}, x_{n-1}\right\} \geq 1 \quad \forall n \geq N .
$$

That is, there exists a positive real number $m$ such that

$$
x_{n} \geq m \quad \forall n \geq N .
$$

Thus from (1.1), we see that

$$
\begin{aligned}
x_{n+1} & =\max \left\{\frac{1}{x_{n}}, \frac{A_{n}}{x_{n-1}}\right\} \\
& \leq \max \left\{\frac{1}{m}, \frac{A_{n}}{m}\right\}=M .
\end{aligned}
$$

Hence

$$
x_{n} \leq M \quad \forall n \geq N .
$$

Thus from inequalities (2.4) and (2.6) we get

$$
0<m \leq x_{n} \leq M<\infty \quad \forall n \geq N .
$$

Therefore every solution of (1.1) is bounded and persists.

Lemma 2.2. Assume that $\left\{x_{n}\right\}_{n=-1}^{\infty}$ is a positive solution of (1.1). Suppose there exists $N \geq 0$ such that

$$
x_{N-1}, x_{N} \in\left[\frac{1}{\sqrt{\alpha}}, \beta \sqrt{\alpha}\right] \text { for some } N \geq 0 \text {. }
$$

Then

$$
x_{n} \in\left[\frac{\sqrt{\alpha}}{\beta}, \beta \sqrt{\alpha}\right] \quad \forall n \geq N .
$$

Proof. Observe from (1.1) that

$$
\begin{gathered}
x_{N+1}=\max \left\{\frac{1}{x_{N}}, \frac{A_{N}}{x_{N-1}}\right\} \geq \max \left\{\frac{1}{\beta \sqrt{\alpha}}, \frac{\alpha}{\beta \sqrt{\alpha}}\right\}=\frac{\sqrt{\alpha}}{\beta}, \\
x_{N+1} \leq \max \{\sqrt{\alpha}, \alpha \sqrt{\alpha}\}=\alpha \sqrt{\alpha}<\beta \sqrt{\alpha} .
\end{gathered}
$$


Then

$$
\frac{\sqrt{\alpha}}{\beta} \leq x_{N+1}<\beta \sqrt{\alpha} .
$$

Again

$$
\begin{gathered}
x_{N+2}=\max \left\{\frac{1}{x_{N+1}}, \frac{A_{N+1}}{x_{N}}\right\} \geq \max \left\{\frac{1}{\beta \sqrt{\alpha}}, \frac{\beta}{\beta \sqrt{\alpha}}\right\}=\frac{1}{\sqrt{\alpha}}, \\
x_{N+2} \leq \max \left\{\frac{\beta}{\sqrt{\alpha}}, \beta \sqrt{\alpha}\right\}=\beta \sqrt{\alpha} .
\end{gathered}
$$

Then

$$
\frac{1}{\sqrt{\alpha}} \leq x_{N+2} \leq \beta \sqrt{\alpha}
$$

Also we see from (1.1) that

$$
\begin{gathered}
x_{N+3}=\max \left\{\frac{1}{x_{N+2}}, \frac{A_{N+2}}{x_{N+1}}\right\} \geq \max \left\{\frac{1}{\beta \sqrt{\alpha}}, \frac{\alpha}{\alpha \sqrt{\alpha}}\right\}=\frac{1}{\sqrt{\alpha}}, \\
x_{N+3} \leq \max \{\sqrt{\beta}, \beta \sqrt{\alpha}\}=\beta \sqrt{\alpha} .
\end{gathered}
$$

Then

$$
\frac{1}{\sqrt{\alpha}} \leq x_{N+3} \leq \beta \sqrt{\alpha} .
$$

Thus following the above procedure we have

$$
x_{n} \in\left[\frac{\sqrt{\alpha}}{\beta}, \beta \sqrt{\alpha}\right] \quad \forall n \geq N .
$$

The proof is complete.

Lemмa 2.3. Every solution of (1.1) which is bounded below by $1 / \sqrt{\alpha}$ lies in the interval $[1 / \sqrt{\alpha}, \beta \sqrt{\alpha}]$.

Proof. Let $\left\{x_{n}\right\}_{n=-1}^{\infty}$ be a positive solution of (1.1) and there exists $N \geq 0$ such that

$$
x_{n-1} \geq \frac{1}{\sqrt{\alpha}} \quad \forall n \geq N \text {. }
$$

It follows from (1.1) that

$$
x_{N+1}=\max \left\{\frac{1}{x_{N}}, \frac{A_{N}}{x_{N-1}}\right\} \leq \max \left\{\sqrt{\alpha}, \sqrt{\alpha} A_{N}\right\} \leq \beta \sqrt{\alpha} .
$$

Similarly, we see that

$$
x_{N+2}=\max \left\{\frac{1}{x_{N+1}}, \frac{A_{N+1}}{x_{N}}\right\} \leq \max \left\{\sqrt{\alpha}, \sqrt{\alpha} A_{N+1}\right\} \leq \beta \sqrt{\alpha} .
$$

The rest of the proof follows by Lemma 2.2. 


\section{The main result}

In this section, we study the periodicity character of solutions of (1.1).

In the following we study the existence of periodic solutions of (1.1) with period four.

Theorem 3.1. Assume that $\left\{x_{n}\right\}_{n=-1}^{\infty}$ is a positive solution of (1.1) with

$$
\frac{1}{\sqrt{\alpha}}<x_{N-1}, \quad x_{N}<\sqrt{\beta}
$$

Then $\left\{x_{n}\right\}_{n=-1}^{\infty}$ is a four-cycle solution of (1.1).

Proof. Let $\left\{x_{n}\right\}_{n=-1}^{\infty}$ be a positive solution of (1.1). Suppose there exists $N \geq 0$ such that

$$
\frac{1}{\sqrt{\alpha}}<x_{N-1}, \quad x_{N}<\sqrt{\beta} .
$$

Assume that

$$
x_{N-1}=p, \quad x_{N}=q .
$$

Observe from (1.1) that

$$
x_{N+1}=\max \left\{\frac{1}{x_{N}}, \frac{A_{N}}{x_{N-1}}\right\} .
$$

We consider the following two cases.

(1) $x_{N+1}=1 / x_{N}=1 / q$. In this case $1 / x_{N}>\alpha / x_{N-1}$, (the case $1 / x_{N}>\beta / x_{N-1}$ can be treated similarly) and we see that

$$
\begin{aligned}
& x_{N+2}=\max \left\{\frac{1}{x_{N+1}}, \frac{A_{N+1}}{x_{N}}\right\}=\max \left\{q, \frac{\beta}{q}\right\}=\frac{\beta}{q}, \\
& x_{N+3}=\max \left\{\frac{1}{x_{N+2}}, \frac{A_{N+2}}{x_{N+1}}\right\}=\max \left\{\frac{q}{\beta}, \alpha q\right\}=\alpha q, \\
& x_{N+4}=\max \left\{\frac{1}{x_{N+3}}, \frac{A_{N+3}}{x_{N+2}}\right\}=\max \left\{\frac{1}{\alpha q}, q\right\}=q, \\
& x_{N+5}=\max \left\{\frac{1}{x_{N+4}}, \frac{A_{N+4}}{x_{N+3}}\right\}=\max \left\{\frac{1}{q}, \frac{1}{q}\right\}=\frac{1}{q}, \\
& x_{N+6}=\max \left\{\frac{1}{x_{N+5}}, \frac{A_{N+5}}{x_{N+4}}\right\}=\max \left\{q, \frac{\beta}{q}\right\}=\frac{\beta}{q} .
\end{aligned}
$$


Then clearly the solution becomes in the form

$$
\left\{\ldots, q, \frac{1}{q}, \frac{\beta}{q}, \alpha q, q, \frac{1}{q}, \frac{\beta}{q}, \alpha q, \ldots\right\}
$$

(2) $x_{N+1}=\alpha / x_{N-1}=\alpha / p$. In this case we see that

$$
\begin{aligned}
& x_{N+2}=\max \left\{\frac{1}{x_{N+1}}, \frac{A_{N+1}}{x_{N}}\right\}=\max \left\{\frac{p}{\alpha}, \frac{\beta}{q}\right\}=\frac{\beta}{q}, \\
& x_{N+3}=\max \left\{\frac{1}{x_{N+2}}, \frac{A_{N+2}}{x_{N+1}}\right\}=\max \left\{\frac{q}{\beta}, p\right\}=p,
\end{aligned}
$$

where $x_{N-1}>1 / \sqrt{\beta} \Rightarrow \beta x_{N-1}>\sqrt{\beta}>x_{N}$,

$$
\begin{aligned}
& x_{N+4}=\max \left\{\frac{1}{x_{N+3}}, \frac{A_{N+3}}{x_{N+2}}\right\}=\max \left\{\frac{1}{p}, q\right\}=q, \\
& x_{N+5}=\max \left\{\frac{1}{x_{N+4}}, \frac{A_{N+4}}{x_{N+3}}\right\}=\max \left\{\frac{1}{q}, \frac{\alpha}{p}\right\}=\frac{\alpha}{p}, \\
& x_{N+6}=\max \left\{\frac{1}{x_{N+5}}, \frac{A_{N+5}}{x_{N+4}}\right\}=\max \left\{\frac{p}{\alpha}, \frac{\beta}{q}\right\}=\frac{\beta}{q},
\end{aligned}
$$

and so the solution becomes in the form

$$
\left\{\ldots, p, q, \frac{\alpha}{p}, \frac{\beta}{q}, p, q, \frac{\alpha}{p}, \frac{\beta}{q}, \ldots\right\}
$$

The proof is complete.

THeOREM 3.2. Every positive solution of (1.1) which is bounded from below by $1 / \sqrt{\alpha}$ is eventually periodic with period four.

Proof. Let $\left\{x_{n}\right\}_{n=-1}^{\infty}$ be a positive solution of (1.1). By Lemma 2.3, we assume

$$
\frac{1}{\sqrt{\alpha}}<x_{N-1}, \quad x_{N}<\beta \sqrt{\alpha} \text { for some integer } N \geq 2 .
$$

From (1.1), we see that

$$
x_{N+1}=\max \left\{\frac{1}{x_{N}}, \frac{\alpha}{x_{N-1}}\right\} .
$$

We consider the following two cases.

$\left(A_{1}\right) x_{N+1}=1 / x_{N}$. In this case $1 / x_{N}>\alpha / x_{N-1}$, and we see that

$$
x_{N+2}=\max \left\{\frac{1}{x_{N+1}}, \frac{A_{N+1}}{x_{N}}\right\}=\max \left\{x_{N}, \frac{\beta}{x_{N}}\right\} .
$$

We consider the following two cases. 


\section{Periodicity of max-type difference equations}

$\left(A_{11}\right) x_{N+2}=x_{N}$. In this case $x_{N}>\beta / x_{N}$, and we see that

$$
x_{N+3}=\max \left\{\frac{1}{x_{N+2}}, \frac{A_{N+2}}{x_{N+1}}\right\}=\max \left\{\frac{1}{x_{N}}, \alpha x_{N}\right\}=\alpha x_{N},
$$

where $x_{N}>1 / \sqrt{\alpha} \Rightarrow \alpha x_{N}>1 / x_{N}$,

$$
\begin{aligned}
& x_{N+4}=\max \left\{\frac{1}{x_{N+3}}, \frac{A_{N+3}}{x_{N+2}}\right\}=\max \left\{\frac{1}{\alpha x_{N}}, \frac{\beta}{x_{N}}\right\}=\frac{\beta}{x_{N}}, \\
& x_{N+5}=\max \left\{\frac{1}{x_{N+4}}, \frac{A_{N+4}}{x_{N+3}}\right\}=\max \left\{\frac{x_{N}}{\beta}, \frac{1}{x_{N}}\right\}=\frac{x_{N}}{\beta}, \\
& x_{N+6}=\max \left\{\frac{1}{x_{N+5}}, \frac{A_{N+5}}{x_{N+4}}\right\}=\max \left\{\frac{\beta}{x_{N}}, x_{N}\right\}=x_{N}, \\
& x_{N+7}=\max \left\{\frac{1}{x_{N+6}}, \frac{A_{N+6}}{x_{N+5}}\right\}=\max \left\{\frac{1}{x_{N}}, \frac{\alpha \beta}{x_{N}}\right\}=\frac{\alpha \beta}{x_{N}}, \\
& x_{N+8}=\max \left\{\frac{1}{x_{N+7}}, \frac{A_{N+7}}{x_{N+6}}\right\}=\max \left\{\frac{x_{N}}{\alpha \beta}, \frac{\beta}{x_{N}}\right\}=\frac{\beta}{x_{N}}, \\
& x_{N+10}=\max \left\{\frac{1}{x_{N+9}}, \frac{A_{N+9}}{x_{N+8}}\right\}=\max \left\{\frac{1}{x_{N+8}}, \frac{A_{N+8}}{x_{N+7}}\right\}=\max \left\{\frac{x_{N}}{\beta}, \frac{x_{N}}{\beta}\right\}=\frac{x_{N}}{\beta}, \\
& x_{N+11}=\max \left\{\frac{1}{x_{N+10}}, \frac{A_{N+10}}{x_{N+9}}\right\}=\max \left\{\frac{1}{x_{N}}, \frac{\alpha \beta}{x_{N}}\right\}=\frac{\alpha \beta}{x_{N}}, \\
& x_{N+12}=\max \left\{\frac{1}{x_{N+11}}, \frac{A_{N+11}}{x_{N+10}}\right\}=\max \left\{\frac{x_{N}}{\alpha \beta}, \frac{\beta}{x_{N}}\right\}=\frac{\beta}{x_{N}} .
\end{aligned}
$$

We see that the solution is in the form

$$
\left\{\ldots, \frac{\beta}{x_{N}}, \frac{x_{N}}{\beta}, x_{N}, \frac{\alpha \beta}{x_{N}}, \frac{\beta}{x_{N}}, \frac{x_{N}}{\beta}, x_{N}, \frac{\alpha \beta}{x_{N}}, \ldots\right\} .
$$

Therefore $\left\{x_{n}\right\}_{n=-1}^{\infty}$ is a periodic solution with period four.

$\left(A_{12}\right) x_{N+2}=\beta / x_{N}$. In this case $\beta / x_{N}>x_{N}$, and we see that

$$
\begin{aligned}
& x_{N+3}=\max \left\{\frac{1}{x_{N+2}}, \frac{A_{N+2}}{x_{N+1}}\right\}=\max \left\{\frac{x_{N}}{\beta}, \alpha x_{N}\right\}=\alpha x_{N}, \\
& x_{N+4}=\max \left\{\frac{1}{x_{N+3}}, \frac{A_{N+3}}{x_{N+2}}\right\}=\max \left\{\frac{1}{\alpha x_{N}}, x_{N}\right\}=x_{N},
\end{aligned}
$$


where $x_{N}>1 / \sqrt{\alpha} \Rightarrow x_{N}^{2}>1 / \alpha \Rightarrow x_{N}>1 / \alpha x_{N}$,

$$
\begin{aligned}
& x_{N+5}=\max \left\{\frac{1}{x_{N+4}}, \frac{A_{N+4}}{x_{N+3}}\right\}=\max \left\{\frac{1}{x_{N}}, \frac{1}{x_{N}}\right\}=\frac{1}{x_{N}}, \\
& x_{N+6}=\max \left\{\frac{1}{x_{N+5}}, \frac{A_{N+5}}{x_{N+4}}\right\}=\max \left\{x_{N}, \frac{\beta}{x_{N}}\right\}=\frac{\beta}{x_{N}} \text {, } \\
& x_{N+7}=\max \left\{\frac{1}{x_{N+6}}, \frac{A_{N+6}}{x_{N+5}}\right\}=\max \left\{\frac{x_{N}}{\beta}, \alpha x_{N}\right\}=\alpha x_{N} \text {, } \\
& x_{N+8}=\max \left\{\frac{1}{x_{N+7}}, \frac{A_{N+7}}{x_{N+6}}\right\}=\max \left\{\frac{1}{\alpha x_{N}}, x_{N}\right\}=x_{N}, \\
& x_{N+9}=\max \left\{\frac{1}{x_{N+8}}, \frac{A_{N+8}}{x_{N+7}}\right\}=\max \left\{\frac{1}{x_{N}}, \frac{1}{x_{N}}\right\}=\frac{1}{x_{N}} \text {, } \\
& x_{N+10}=\max \left\{\frac{1}{x_{N+9}}, \frac{A_{N+9}}{x_{N+8}}\right\}=\max \left\{x_{N}, \frac{\beta}{x_{N}}\right\}=\frac{\beta}{x_{N}}, \\
& x_{N+11}=\max \left\{\frac{1}{x_{N+10}}, \frac{A_{N+10}}{x_{N+9}}\right\}=\max \left\{\frac{x_{N}}{\beta}, \alpha x_{N}\right\}=\alpha x_{N} \text {, } \\
& x_{N+12}=\max \left\{\frac{1}{x_{N+11}}, \frac{A_{N+11}}{x_{N+10}}\right\}=\max \left\{\frac{1}{\alpha x_{N}}, x_{N}\right\}=x_{N} \text {. }
\end{aligned}
$$

Therefore $\left\{x_{n}\right\}_{n=-1}^{\infty}$ is a periodic solution with period four as follows:

$$
\left\{\ldots, x_{N}, \frac{1}{x_{N}}, \frac{\beta}{x_{N}}, \alpha x_{N}, x_{N}, \frac{1}{x_{N}}, \frac{\beta}{x_{N}}, \alpha x_{N}, \ldots\right\} \text {. }
$$

$\left(A_{2}\right) x_{N+1}=\alpha / x_{N-1}$. In this case $\alpha / x_{N-1}>1 / x_{N}$, and we see that

$$
x_{N+2}=\max \left\{\frac{1}{x_{N+1}}, \frac{A_{N+1}}{x_{N}}\right\}=\max \left\{\frac{x_{N-1}}{\alpha}, \frac{\beta}{x_{N}}\right\} .
$$

We consider the following two cases.

$\left(A_{21}\right) x_{N+2}=x_{N-1} / \alpha$. In this case $x_{N-1} / \alpha>\beta / x_{N}$, and we see that

$$
x_{N+3}=\max \left\{\frac{1}{x_{N+2}}, \frac{A_{N+2}}{x_{N+1}}\right\}=\max \left\{\frac{\alpha}{x_{N-1}}, x_{N-1}\right\}=x_{N-1},
$$

where $\beta \sqrt{\alpha} x_{N-1}>x_{N-1} x_{N}>\alpha \beta \Rightarrow x_{N-1}>\sqrt{\alpha}$,

$$
\begin{aligned}
& x_{N+4}=\max \left\{\frac{1}{x_{N+3}}, \frac{A_{N+3}}{x_{N+2}}\right\}=\max \left\{\frac{1}{x_{N-1}}, \frac{\alpha \beta}{x_{N-1}}\right\}=\frac{\alpha \beta}{x_{N-1}}, \\
& x_{N+5}=\max \left\{\frac{1}{x_{N+4}}, \frac{A_{N+4}}{x_{N+3}}\right\}=\max \left\{\frac{x_{N-1}}{\alpha \beta}, \frac{\alpha}{x_{N-1}}\right\}=\frac{x_{N-1}}{\alpha \beta} .
\end{aligned}
$$

We consider the following two cases.

$\left(A_{211}\right) x_{N+5}=x_{N-1} / \alpha \beta$. In this case $x_{N-1} / \alpha \beta>\alpha / x_{N-1}$, and we see that

$$
x_{N+6}=\max \left\{\frac{1}{x_{N+5}}, \frac{A_{N+5}}{x_{N+4}}\right\}=\max \left\{\frac{\alpha \beta}{x_{N-1}}, \frac{x_{N-1}}{\alpha}\right\}=\frac{x_{N-1}}{\alpha},
$$


where $x_{N-1} / \alpha \beta>\alpha / x_{N-1} \Rightarrow x_{N-1} / \alpha>\alpha \beta / x_{N-1}$,

$$
\begin{aligned}
& x_{N+7}=\max \left\{\frac{1}{x_{N+6}}, \frac{A_{N+6}}{x_{N+5}}\right\}=\max \left\{\frac{\alpha}{x_{N-1}}, \frac{\alpha^{2} \beta}{x_{N-1}}\right\}=\frac{\alpha^{2} \beta}{x_{N-1}}, \\
& x_{N+8}=\max \left\{\frac{1}{x_{N+7}}, \frac{A_{N+7}}{x_{N+6}}\right\}=\max \left\{\frac{x_{N-1}}{\alpha^{2} \beta}, \frac{\alpha \beta}{x_{N-1}}\right\}=\frac{\alpha \beta}{x_{N-1}},
\end{aligned}
$$

where $x_{N-1}<\beta \sqrt{\alpha} \Rightarrow x_{N-1}^{2}<\beta^{2} \alpha<\beta^{2} \alpha^{3} \Rightarrow \alpha \beta / x_{N-1}>x_{N-1} / \alpha^{2} \beta$,

$$
\begin{aligned}
& x_{N+9}=\max \left\{\frac{1}{x_{N+8}}, \frac{A_{N+8}}{x_{N+7}}\right\}=\max \left\{\frac{x_{N-1}}{\alpha \beta}, \frac{x_{N-1}}{\alpha \beta}\right\}=\frac{x_{N-1}}{\alpha \beta}, \\
& x_{N+10}=\max \left\{\frac{1}{x_{N+9}}, \frac{A_{N+9}}{x_{N+8}}\right\}=\max \left\{\frac{\alpha \beta}{x_{N-1}}, \frac{x_{N-1}}{\alpha}\right\}=\frac{x_{N-1}}{\alpha}, \\
& x_{N+11}=\max \left\{\frac{1}{x_{N+10}}, \frac{A_{N+10}}{x_{N+9}}\right\}=\max \left\{\frac{\alpha}{x_{N-1}}, \frac{\alpha^{2} \beta}{x_{N-1}}\right\}=\frac{\alpha^{2} \beta}{x_{N-1}}, \\
& x_{N+12}=\max \left\{\frac{1}{x_{N+11}}, \frac{A_{N+11}}{x_{N+10}}\right\}=\max \left\{\frac{x_{N-1}}{\alpha^{2} \beta}, \frac{\alpha \beta}{x_{N-1}}\right\}=\frac{\alpha \beta}{x_{N-1}} .
\end{aligned}
$$

Therefore the solution can be written as

$$
\left\{\ldots, \frac{\alpha \beta}{x_{N-1}}, \frac{x_{N-1}}{\alpha \beta}, \frac{x_{N-1}}{\alpha}, \frac{\alpha^{2} \beta}{x_{N-1}}, \frac{\alpha \beta}{x_{N-1}}, \frac{x_{N-1}}{\alpha \beta}, \frac{x_{N-1}}{\alpha}, \frac{\alpha^{2} \beta}{x_{N-1}}, \ldots\right\} .
$$

Then $\left\{x_{n}\right\}_{n=-1}^{\infty}$ is a periodic solution with period four.

We consider the following two cases.

$\left(A_{212}\right) x_{N+5}=\alpha / x_{N-1}$. In this case $\alpha / x_{N-1}>x_{N-1} / \alpha \beta$, and we see that

$$
\begin{aligned}
& x_{N+6}=\max \left\{\frac{1}{x_{N+5}}, \frac{A_{N+5}}{x_{N+4}}\right\}=\max \left\{\frac{x_{N-1}}{\alpha}, \frac{x_{N-1}}{\alpha}\right\}=\frac{x_{N-1}}{\alpha}, \\
& x_{N+7}=\max \left\{\frac{1}{x_{N+6}}, \frac{A_{N+6}}{x_{N+5}}\right\}=\max \left\{\frac{\alpha}{x_{N-1}}, x_{N-1}\right\}=x_{N-1}, \\
& x_{N+8}=\max \left\{\frac{1}{x_{N+7}}, \frac{A_{N+7}}{x_{N+6}}\right\}=\max \left\{\frac{1}{x_{N-1}}, \frac{\alpha \beta}{x_{N-1}}\right\}=\frac{\alpha \beta}{x_{N-1}}, \\
& x_{N+9}=\max \left\{\frac{1}{x_{N+8}}, \frac{A_{N+8}}{x_{N+7}}\right\}=\max \left\{\frac{x_{N-1}}{\alpha \beta}, \frac{\alpha}{x_{N-1}}\right\}=\frac{\alpha}{x_{N-1}}, \\
& x_{N+10}=\max \left\{\frac{1}{x_{N+9}}, \frac{A_{N+9}}{x_{N+8}}\right\}=\max \left\{\frac{x_{N-1}}{\alpha}, \frac{x_{N-1}}{\alpha}\right\}=\frac{x_{N-1}}{\alpha}, \\
& x_{N+11}=\max \left\{\frac{1}{x_{N+10}}, \frac{A_{N+10}}{x_{N+9}}\right\}=\max \left\{\frac{\alpha}{x_{N-1}}, x_{N-1}\right\}=x_{N-1}, \\
& x_{N+12}=\max \left\{\frac{1}{x_{N+11}}, \frac{A_{N+11}}{x_{N+10}}\right\}=\max \left\{\frac{1}{x_{N-1}}, \frac{\alpha \beta}{x_{N-1}}\right\}=\frac{\alpha \beta}{x_{N-1}} .
\end{aligned}
$$


It is also easy to see that the solution takes the form

$$
\left\{\ldots, \frac{\alpha}{x_{N-1}}, \frac{x_{N-1}}{\alpha}, x_{N-1}, \frac{\alpha \beta}{x_{N-1}}, \frac{\alpha}{x_{N-1}}, \frac{x_{N-1}}{\alpha}, x_{N-1}, \frac{\alpha \beta}{x_{N-1}}, \ldots\right\}
$$

which is periodic with period four.

$\left(A_{22}\right) x_{N+2}=\beta / x_{N}$. In this case $\beta / x_{N}>x_{N-1} / \alpha$, and we see that

$$
x_{N+3}=\max \left\{\frac{1}{x_{N+2}}, \frac{A_{N+2}}{x_{N+1}}\right\}=\max \left\{\frac{x_{N}}{\beta}, x_{N-1}\right\} .
$$

We consider the following two cases.

$\left(A_{221}\right) x_{N+3}=x_{N-1}$. In this case $x_{N-1}>x_{N} / \beta$, and we see that

$$
\begin{aligned}
& x_{N+4}=\max \left\{\frac{1}{x_{N+3}}, \frac{A_{N+3}}{x_{N+2}}\right\}=\max \left\{\frac{1}{x_{N-1}}, x_{N}\right\}=x_{N}, \\
& x_{N+5}=\max \left\{\frac{1}{x_{N+4}}, \frac{A_{N+4}}{x_{N+3}}\right\}=\max \left\{\frac{1}{x_{N}}, \frac{\alpha}{x_{N-1}}\right\}=\frac{\alpha}{x_{N-1}}, \\
& x_{N+6}=\max \left\{\frac{1}{x_{N+5}}, \frac{A_{N+5}}{x_{N+4}}\right\}=\max \left\{\frac{x_{N-1}}{\alpha}, \frac{\beta}{x_{N}}\right\}=\frac{\beta}{x_{N}}, \\
& x_{N+7}=\max \left\{\frac{1}{x_{N+6}}, \frac{A_{N+6}}{x_{N+5}}\right\}=\max \left\{\frac{x_{N}}{\beta}, x_{N-1}\right\}=x_{N-1}, \\
& x_{N+8}=\max \left\{\frac{1}{x_{N+7}}, \frac{A_{N+7}}{x_{N+6}}\right\}=\max \left\{\frac{1}{x_{N-1}}, x_{N}\right\}=x_{N}, \\
& x_{N+9}=\max \left\{\frac{1}{x_{N+8}}, \frac{A_{N+8}}{x_{N+7}}\right\}=\max \left\{\frac{1}{x_{N}}, \frac{\alpha}{x_{N-1}}\right\}=\max \left\{\frac{1}{x_{N+9}}, \frac{A_{N+9}}{x_{N+8}}\right\}=\max \left\{\frac{x_{N-1}}{\alpha}, \frac{\beta}{x_{N}}\right\}=\frac{\beta}{x_{N}}, \\
& x_{N+11}=\max \left\{\frac{1}{x_{N+10}}, \frac{A_{N+10}}{x_{N+9}}\right\}=\max \left\{\frac{x_{N}}{\beta}, x_{N-1}\right\}=x_{N-1}, \\
& x_{N+12}=\max \left\{\frac{1}{x_{N+11}}, \frac{A_{N+11}}{x_{N+10}}\right\}=\max \left\{\frac{1}{x_{N-1}}, x_{N}\right\}=x_{N} .
\end{aligned}
$$

One can easily see that the solution will be in the form

$$
\left\{\ldots, x_{N-1}, x_{N}, \frac{\alpha}{x_{N-1}}, \frac{\beta}{x_{N}}, x_{N-1}, x_{N}, \frac{\alpha}{x_{N-1}}, \frac{\beta}{x_{N}}, \ldots\right\}
$$

and so the solution is periodic with period four. 
2236 Periodicity of max-type difference equations

$\left(A_{222}\right) x_{N+3}=x_{N} / \beta$. In this case $x_{N} / \beta>x_{N-1}$, and we see that

$$
x_{N+4}=\max \left\{\frac{1}{x_{N+3}}, \frac{A_{N+3}}{x_{N+2}}\right\}=\max \left\{\frac{\beta}{x_{N}}, x_{N}\right\}=x_{N},
$$

where $x_{N}>\beta x_{N-1}>\beta / \sqrt{\alpha}>\beta / \sqrt{\beta}=\sqrt{\beta} \Rightarrow x_{N}^{2}>\beta \Rightarrow x_{N}>\beta / x_{N}$,

$$
\begin{aligned}
& x_{N+5}=\max \left\{\frac{1}{x_{N+4}}, \frac{A_{N+4}}{x_{N+3}}\right\}=\max \left\{\frac{1}{x_{N}}, \frac{\alpha \beta}{x_{N}}\right\}=\frac{\alpha \beta}{x_{N}}, \\
& x_{N+6}=\max \left\{\frac{1}{x_{N+5}}, \frac{A_{N+5}}{x_{N+4}}\right\}=\max \left\{\frac{x_{N}}{\alpha \beta}, \frac{\beta}{x_{N}}\right\}=\frac{\beta}{x_{N}},
\end{aligned}
$$

where $x_{N}<\beta \sqrt{\alpha}$,

$$
\begin{aligned}
& x_{N+7}=\max \left\{\frac{1}{x_{N+6}}, \frac{A_{N+6}}{x_{N+5}}\right\}=\max \left\{\frac{x_{N}}{\beta}, \frac{x_{N}}{\beta}\right\}=\frac{x_{N}}{\beta}, \\
& x_{N+8}=\max \left\{\frac{1}{x_{N+7}}, \frac{A_{N+7}}{x_{N+6}}\right\}=\max \left\{\frac{\beta}{x_{N}}, x_{N}\right\}=x_{N}, \\
& x_{N+9}=\max \left\{\frac{1}{x_{N+8}}, \frac{A_{N+8}}{x_{N+7}}\right\}=\max \left\{\frac{1}{x_{N}}, \frac{\alpha \beta}{x_{N}}\right\}=\frac{\alpha \beta}{x_{N}}, \\
& x_{N+10}=\max \left\{\frac{1}{x_{N+9}}, \frac{A_{N+9}}{x_{N+8}}\right\}=\max \left\{\frac{x_{N}}{\alpha \beta}, \frac{\beta}{x_{N}}\right\}=\frac{\beta}{x_{N}}, \\
& x_{N+11}=\max \left\{\frac{1}{x_{N+10}}, \frac{A_{N+10}}{x_{N+9}}\right\}=\max \left\{\frac{x_{N}}{\beta}, \frac{x_{N}}{\beta}\right\}=\frac{x_{N}}{\beta}, \\
& x_{N+12}=\max \left\{\frac{1}{x_{N+11}}, \frac{A_{N+11}}{x_{N+10}}\right\}=\max \left\{\frac{\beta}{x_{N}}, x_{N}\right\}=x_{N} .
\end{aligned}
$$

Then the solution can be written in the form

$$
\left\{\ldots, \frac{\beta}{x_{N}}, \frac{x_{N}}{\beta}, x_{N}, \frac{\alpha \beta}{x_{N}}, \frac{\beta}{x_{N}}, \frac{x_{N}}{\beta}, x_{N}, \frac{\alpha \beta}{x_{N}}, \ldots\right\},
$$

and so the solution is periodic with period four.

This completes the proof. The proof of Theorem 3.2 is thus completed.

Lemma 3.3. Assume $\left\{x_{n}\right\}_{n=-1}^{\infty}$ is a positive solution of (1.1) and suppose there exists $m \geq 2$ such that

$$
x_{m}<\frac{1}{\sqrt{\alpha}}<x_{m+1} .
$$

Then either $\left\{x_{n}\right\}_{n=-1}^{\infty}$ is eventually periodic solution with period four or

$$
\liminf _{n \rightarrow \infty} x_{n} \geq \frac{1}{\sqrt{\alpha}}
$$


Proof. Observe that $x_{m}<1 / \sqrt{\alpha}$ and either $x_{m+1}<\beta \sqrt{\alpha}$ or $x_{m+1}>\beta \sqrt{\alpha}$.

(i) Assume that $x_{m+1}<\beta \sqrt{\alpha}$. It follows from (1.1) that

$$
x_{m+2}=\max \left\{\frac{1}{x_{m+1}}, \frac{A_{m+1}}{x_{m}}\right\}=\max \left\{\frac{1}{x_{m+1}}, \frac{\alpha}{x_{m}}\right\}=\frac{\alpha}{x_{m}},
$$

where $x_{m} x_{m+1} \geq 1 \Rightarrow x_{m+1}>\sqrt{\alpha}>1$,

$$
x_{m+3}=\max \left\{\frac{1}{x_{m+2}}, \frac{A_{m+2}}{x_{m+1}}\right\}=\max \left\{\frac{x_{m}}{\alpha}, \frac{\beta}{x_{m+1}}\right\}=\frac{\beta}{x_{m+1}},
$$

where $x_{m} x_{m+1}<\beta \sqrt{\alpha} / \sqrt{\alpha}=\beta<\alpha \beta$, and

$$
x_{m+4}=\max \left\{\frac{1}{x_{m+3}}, \frac{A_{m+3}}{x_{m+2}}\right\}=\max \left\{\frac{x_{m+1}}{\beta}, x_{m}\right\} .
$$

Then either

$$
x_{m+4}=\frac{x_{m+1}}{\beta} \quad \text { or } \quad x_{m+4}=x_{m}
$$

and by simple computations the solution becomes either

$$
\left\{\ldots, \frac{x_{m+1}}{\beta}, x_{m+1}, \frac{\alpha \beta}{x_{m+1}}, \frac{\beta}{x_{m+1}}, \frac{x_{m+1}}{\beta}, x_{m+1}, \frac{\alpha \beta}{x_{m+1}}, \frac{\beta}{x_{m+1}}, \ldots\right\},
$$

or

$$
\left\{\ldots, x_{m}, x_{m+1}, \frac{\alpha}{x_{m}}, \frac{\beta}{x_{m+1}}, x_{m}, x_{m+1}, \frac{\alpha}{x_{m}}, \frac{\beta}{x_{m+1}}, \ldots\right\}
$$

and so in either case $\left\{x_{n}\right\}_{n=-1}^{\infty}$ is a periodic solution with period four.

(ii) Assume that $x_{m+1}>\beta \sqrt{\alpha}$. In this case we see from (1.1) that

$$
\begin{gathered}
x_{m+2}=\max \left\{\frac{1}{x_{m+1}}, \frac{A_{m+1}}{x_{m}}\right\}=\max \left\{\frac{1}{x_{m+1}}, \frac{\alpha}{x_{m}}\right\}=\frac{\alpha}{x_{m}}, \\
x_{m+3}=\max \left\{\frac{1}{x_{m+2}}, \frac{A_{m+2}}{x_{m+1}}\right\}=\max \left\{\frac{x_{m}}{\alpha}, \frac{\beta}{x_{m+1}}\right\} .
\end{gathered}
$$

We consider the following two cases. 
(B $B_{1} x_{m+3}=x_{m} / \alpha$. In this case we see that

$$
\begin{aligned}
& x_{m+4}=\max \left\{\frac{1}{x_{m+3}}, \frac{A_{m+3}}{x_{m+2}}\right\}=\max \left\{\frac{\alpha}{x_{m}}, x_{m}\right\}=\frac{\alpha}{x_{m}}, \\
& x_{m+5}=\max \left\{\frac{1}{x_{m+4}}, \frac{A_{m+4}}{x_{m+3}}\right\}=\max \left\{\frac{x_{m}}{\alpha}, \frac{\alpha \beta}{x_{m}}\right\}=\frac{\alpha \beta}{x_{m}}, \\
& x_{m+6}=\max \left\{\frac{1}{x_{m+5}}, \frac{A_{m+5}}{x_{m+4}}\right\}=\max \left\{\frac{x_{m}}{\alpha \beta}, x_{m}\right\}=x_{m}, \\
& x_{m+7}=\max \left\{\frac{1}{x_{m+6}}, \frac{A_{m+6}}{x_{m+5}}\right\}=\max \left\{\frac{1}{x_{m}}, \frac{x_{m}}{\alpha}\right\}=\frac{1}{x_{m}}, \\
& x_{m+8}=\max \left\{\frac{1}{x_{m+7}}, \frac{A_{m+7}}{x_{m+6}}\right\}=\max \left\{x_{m}, \frac{\alpha}{x_{m}}\right\}=\frac{\alpha}{x_{m}}, \\
& x_{m+9}=\max \left\{\frac{1}{x_{m+8}}, \frac{A_{m+8}}{x_{m+7}}\right\}=\max \left\{\frac{x_{m}}{\alpha}, \beta x_{m}\right\}=\beta x_{m}, \\
& x_{m+10}=\max \left\{\frac{1}{x_{m+9}}, \frac{A_{m+9}}{x_{m+8}}\right\}=\max \left\{\frac{1}{\beta x_{m}}, x_{m}\right\} .
\end{aligned}
$$

We consider the following two cases.

$\left(B_{11}\right) x_{m+10}=x_{m}$. In this case the solution eventually will be periodic with period four as

$$
\left\{\ldots, x_{m}, \frac{1}{x_{m}}, \frac{\alpha}{x_{m}}, \beta x_{m}, x_{m}, \frac{1}{x_{m}}, \frac{\alpha}{x_{m}}, \beta x_{m}, \ldots\right\} .
$$

$\left(B_{12}\right) x_{m+10}=1 / \beta x_{m}$. In this case straightforward calculations show that the solution will be in the form

$$
\left\{\ldots, \frac{x_{m}}{\alpha}, \frac{\alpha}{x_{m}}, \frac{\alpha \beta}{x_{m}}, x_{m}, \frac{1}{x_{m}}, \frac{\alpha}{x_{m}}, \beta x_{m}, \frac{1}{\beta x_{m}}, \frac{1}{x_{m}}, \alpha \beta x_{m}, \ldots\right\} .
$$

Thus the subsequence $\left\{x_{m+3 i}\right\}_{i=0}^{\infty}$ is increasing and so

$$
\lim _{i \rightarrow \infty} x_{n+3 i} \geq \frac{1}{\sqrt{\alpha}}
$$

$\left(B_{2}\right) x_{m+3}=\beta / x_{m+1}$. This can be treated similarly to the case $x_{m+3}=x_{m} / \alpha$ and the solution is either periodic with period four or $\lim _{i \rightarrow \infty} x_{n+3 i} \geq 1 / \sqrt{\alpha}$.

The proof is complete.

Remark 3.4. Observe by assumption that $x_{m}, x_{m+1}<1 / \sqrt{\alpha}$ is not possible as can be seen from (1.1).

Now, we can state the main result in this section.

Theorem 3.5. Every solution of (1.1) is periodic with period four.

Proof. The proof of this theorem follows from Theorem 3.2 and Lemma 3.3. 


\section{References}

[1] A. M. Amleh, J. Hoag, and G. Ladas, A difference equation with eventually periodic solutions, Comput. Math. Appl. 36 (1998), no. 10-12, 401-404.

[2] W. J. Briden, E. A. Grove, G. Ladas, and C. M. Kent, Eventually periodic solutions of $x_{n+1}=$ $\max \left\{1 / x_{n}, A_{n} / x_{n-1}\right\}$, Comm. Appl. Nonlinear Anal. 6 (1999), no. 4, 31-43.

[3] W. J. Briden, E. A. Grove, G. Ladas, and L. C. McGrath, On the nonautonomous equation $x_{n+1}=$ $\max \left\{A_{n} / x_{n}, B_{n} / x_{n-1}\right\}$, New Developments in Difference Equations and Applications (Taipei, 1997), Gordon and Breach, Amsterdam, 1999, pp. 49-73.

[4] E. M. Elabbasy, H. El-Metwally, and E. M. Elsayed, On the periodicity behavior of some difference equations, to appear.

[5] H. El-Metwally, E. A. Grove, and G. Ladas, A global convergence result with applications to periodic solutions, J. Math. Anal. Appl. 245 (2000), no. 1, 161-170.

[6] H. El-Metwally, E. A. Grove, G. Ladas, and H. D. Voulov, On the global attractivity and the periodic character of some difference equations, J. Differ. Equations Appl. 7 (2001), no. 6, 837-850.

[7] V. L. Kocić and G. Ladas, Global Behavior of Nonlinear Difference Equations of Higher Order With Applications, Mathematics and Its Applications, vol. 256, Kluwer Academic, Dordrecht, 1993.

[8] S. Stević, A generalization of the Copson's theorem concerning sequences which satisfy a linear inequality, Indian J. Math. 43 (2001), no. 3, 277-282.

E. M. Elabbasy: Department of Mathematics, Faculty of Science, Mansoura University, Mansoura 35516, Egypt

E-mail address: emelabbasy@mans.edu.eg

H. El-Metwally: Department of Mathematics, Faculty of Science, Mansoura University, Mansoura 35516, Egypt

E-mail address: helmetwally@mans.edu.eg

E. M. Elsayed: Department of Mathematics, Faculty of Science, Mansoura University, Mansoura 35516, Egypt

E-mail address: emelsayed@mans.edu.eg 


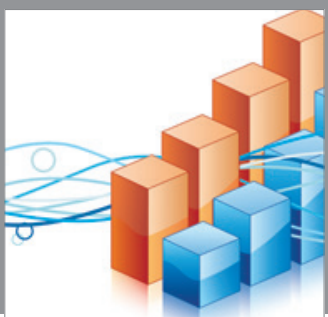

Advances in

Operations Research

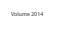

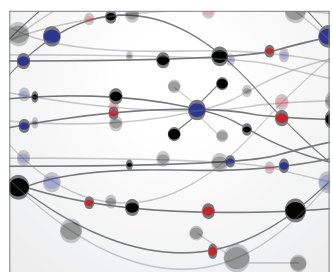

\section{The Scientific} World Journal
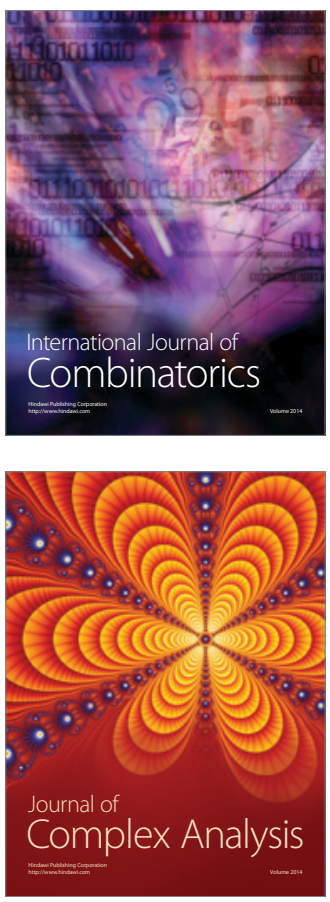

International Journal of

Mathematics and

Mathematical

Sciences
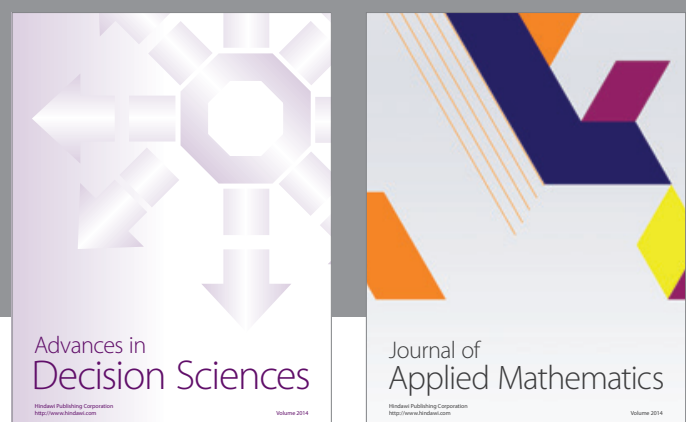

Journal of

Applied Mathematics
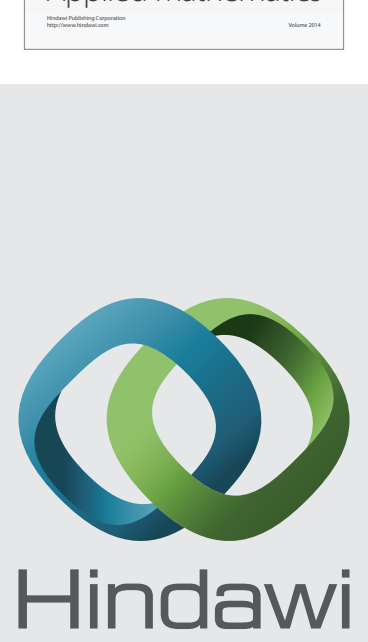

Submit your manuscripts at http://www.hindawi.com
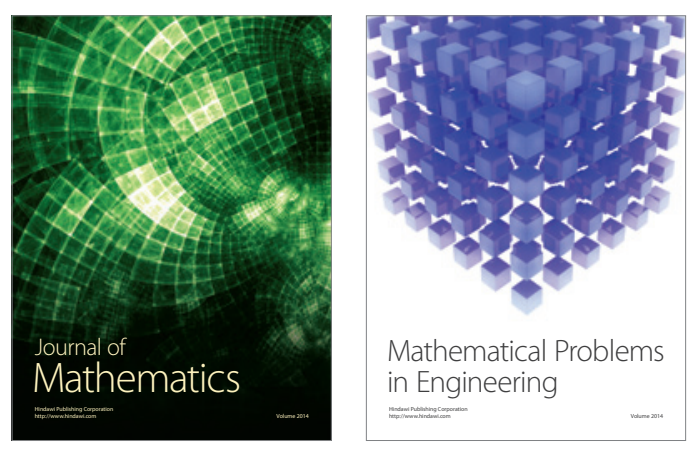

Mathematical Problems in Engineering
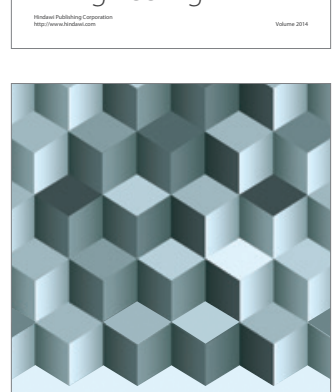

Journal of

Function Spaces
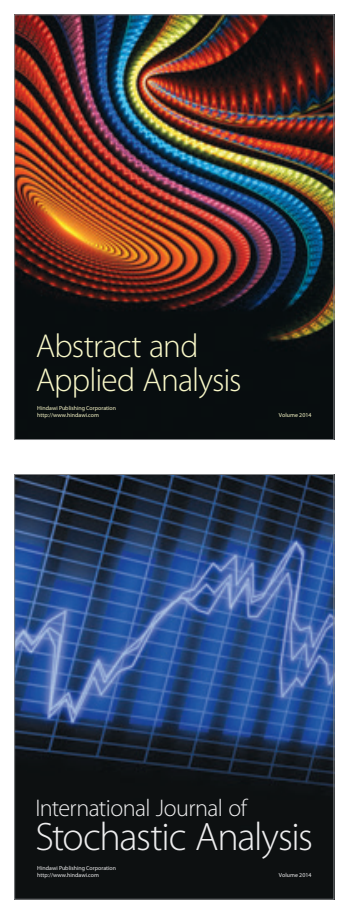

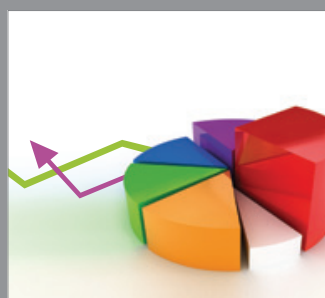

ournal of

Probability and Statistics

Promensencen
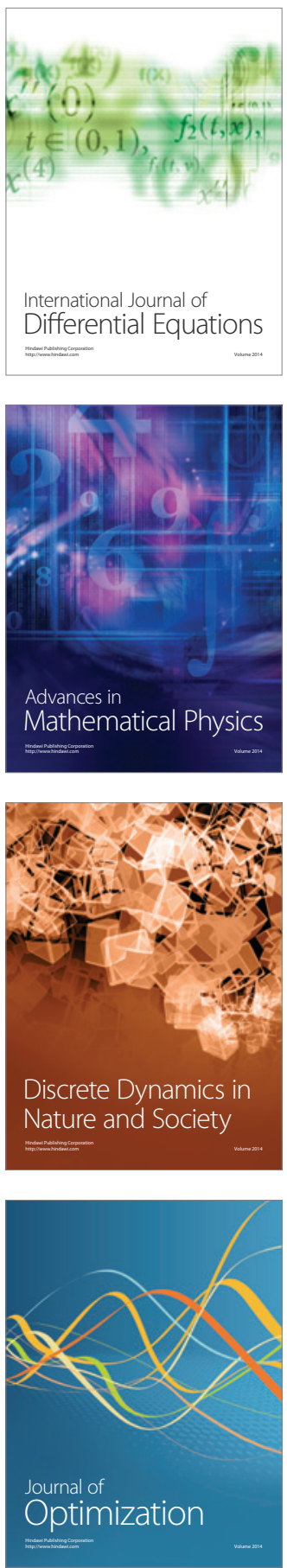